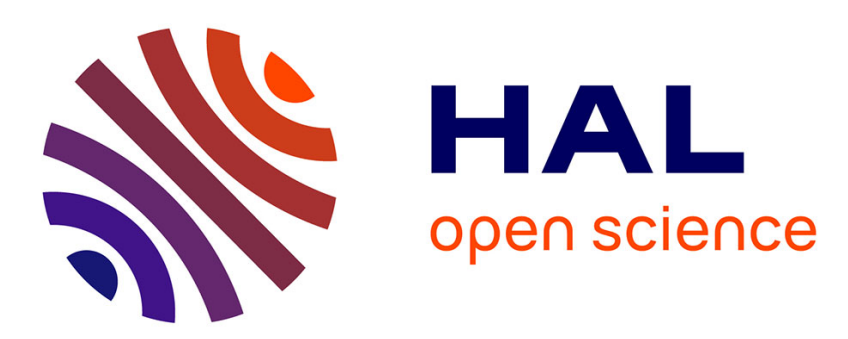

\title{
Estimation and tests of independence in copula models via divergences
}

\author{
Salim Bouzebda, Amor Keziou
}

\section{To cite this version:}

Salim Bouzebda, Amor Keziou. Estimation and tests of independence in copula models via divergences. 41èmes Journées de Statistique, SFdS, Bordeaux, 2009, Bordeaux, France, France. inria00386573

\section{HAL Id: inria-00386573 \\ https://hal.inria.fr/inria-00386573}

Submitted on 22 May 2009

HAL is a multi-disciplinary open access archive for the deposit and dissemination of scientific research documents, whether they are published or not. The documents may come from teaching and research institutions in France or abroad, or from public or private research centers.
L'archive ouverte pluridisciplinaire HAL, est destinée au dépôt et à la diffusion de documents scientifiques de niveau recherche, publiés ou non, émanant des établissements d'enseignement et de recherche français ou étrangers, des laboratoires publics ou privés. 


\title{
ESTIMATION AND TESTS OF INDEPENDENCE IN COPULA MODELS VIA DIVERGENCES
}

\author{
Salim BOUZEBDA ${ }^{1,2}$ and Amor KEZIOU ${ }^{1}$ \\ L.S.T.A., Université Pierre et Marie Curie-Paris 6. 175, rue du Chevaleret, gème étage, \\ bâtiment A,75013 PARIS \\ Transport and Traffic Engineering Laboratory \\ INRETS-ENTEPE, 25 avenue F. Mitterrand \\ 69675 Bron Cedex ${ }^{2}$ \\ salim.bouzebda@upmc.framor.keziou@upmc.fr
}

\begin{abstract}
We introduce new estimates and tests of independence in copula models with unknown margins using $\phi$-divergences and the duality technique. The asymptotic laws of the estimates and the test statistics are established both when the parameter is an interior point or not.
\end{abstract}

\section{Introduction}

Let $F\left(x_{1}, x_{2}\right):=P\left(X_{1} \leq x_{1}, X_{2} \leq x_{2}\right)$ be a 2-dimensional distribution function, and $F_{i}\left(x_{i}\right):=$ $P\left(X_{i} \leq x_{i}\right), i=1,2$ the marginal distribution of $F(\cdot, \cdot)$. It is well known since the work of [14] that there exists a distribution function $C(\cdot, \cdot)$ on $[0,1]^{2}$ with uniform marginals such that

$$
C\left(u_{1}, u_{2}\right):=P\left\{F_{1}\left(X_{1}\right) \leq u_{1}, F_{2}\left(X_{2}\right) \leq u_{2}\right\}
$$

For a systematic theory of copula, see [4, 5, 6] and [11. Many useful multivariate models for dependence between $X_{1}$ and $X_{2}$ turn out to be generated by parametric families of copulas of the form $\left\{C_{\theta} ; \theta \in \Theta\right\}$, typically indexed by a vector valued parameter $\theta \in \Theta \subseteq \mathbb{R}^{d}$ (see, e.g. [11] and [8] among others). In the sequel, we assume that $C_{\theta}(\cdot, \cdot)$ admits a density $c_{\theta}(\cdot, \cdot)$ with respect to the Lebesgue measure $\lambda$ on $\mathbb{R}^{2}$. In this paper, we consider the estimation and test problems for semiparametric copula models with unknown general margins. Let $\left(X_{1 k}, X_{2 k}\right)$ $k=1, \ldots, n$ be a bivariate sample with distribution function $F_{\theta_{T}}(\cdot, \cdot)=C_{\theta_{T}}\left(F_{1}(\cdot), F_{2}(\cdot)\right)$ where $\theta_{T} \in \Theta$ is used to denote the true unknown value of the parameter. In order to estimate $\theta_{T}$, some semiparametric estimation procedures, based on the maximization, on the parameter space $\Theta$, of properly chosen pseudo-likelihood criterion, have been proposed by [12], [16] and [15] among others. In each of these papers, some asymptotic normality properties are established for $\sqrt{n}\left(\tilde{\theta}-\theta_{T}\right)$, where $\tilde{\theta}=\tilde{\theta}_{n}$ denotes a properly chosen estimator of $\theta_{T}$. This is achieved, provided that $\theta_{T}$ lies in the interior, denoted by $\Theta$, of the parameter space $\Theta \subseteq \mathbb{R}^{d}$. On the other hand, the case where $\theta_{T} \in \partial \Theta:=\bar{\Theta}-\dot{\Theta}$ is a boundary value of $\Theta$, has not been studied in a systematical way until present. We find in [9] many examples of parametric copulas, for which marginal independence is verified for some specific values of the parameter $\theta$, on the boundary $\partial \Theta$ of the admissible parameter set $\Theta \subseteq \mathbb{R}^{d}, d \geq 1$. In the sequel, we denote by $\theta_{0}$ the value of the parameter which corresponds to the independence. Moreover, it turns out that, for the above-mentioned estimators, the asymptotic normality of $\sqrt{n}\left(\tilde{\theta}-\theta_{T}\right)$, may fail to hold for $\theta_{T} \in \partial \Theta$. Our approach is novel in this setting and it will become clear later on from our results, that the asymptotic normality of the estimate based on $\phi$-divergences holds, even under the independence assumption, when, either, $\theta_{0}$ is an interior, or a boundary point of $\Theta$. The proposed test statistics of independence using $\phi$ divergences are also studied, under the null hypothesis $\mathscr{H}_{0}$ of independence, as well as under the alternative hypothesis $\mathscr{H}_{1}$. 


\section{A new inference procedure}

Recall that the $\phi$-divergences between a bounded signed measure $Q$, and a probability $P$ on $\mathscr{D}$, when $Q$ is absolutely continuous with respect to $P$, is defined by

$$
D_{\phi}(Q, P):=\int_{\mathscr{D}} \phi\left(\frac{d Q}{d P}\right) d P
$$

where $\phi$ is a proper closed convex function from $]-\infty, \infty[$ to $[0, \infty[$ with $\phi(1)=0$ and such that the domain $\operatorname{dom} \phi:=\{x \in \mathbb{R}: \phi(x)<\infty\}$ is an interval with end points $a_{\phi}<1<b_{\phi}$. The Kullback-Leibler, modified Kullback-Leibler, $\chi^{2}$, modified $\chi^{2}$, Hellinger and $L^{1}$ divergences are examples of $\phi$-divergences; they are obtained respectively for $\phi(x)=x \log x-x+1$, $\phi(x)=-\log x+x-1, \phi(x)=\frac{1}{2}(x-1)^{2}, \phi(x)=\frac{1}{2} \frac{(x-1)^{2}}{x}, \phi(x)=2(\sqrt{x}-1)^{2}$ and $\phi(x)=|x-1|$. We refer to [10] for a systematic theory of divergences. In the sequel, for all $\theta$, we denote by $D_{\phi}\left(\theta, \theta_{T}\right)$ the $\phi$-divergences between $C_{\theta}(\cdot, \cdot)$ and $C_{\theta_{T}}(\cdot, \cdot)$, i.e.,

$$
D_{\phi}\left(\theta, \theta_{T}\right):=\int_{I} \phi\left(\frac{d C_{\theta}}{d C_{\theta_{T}}}\right) d C_{\theta_{T}}\left(u_{1}, u_{2}\right)=\int_{I} \phi\left(\frac{c_{\theta}}{c_{\theta_{T}}}\right) d C_{\theta_{T}}\left(u_{1}, u_{2}\right) .
$$

Denote $C_{n}(\cdot, \cdot)$ the empirical copula associated to the data, i.e.,

$$
C_{n}\left(u_{1}, u_{2}\right):=\frac{1}{n} \sum_{k=1}^{n} \mathbb{1}_{\left\{F_{1 n}\left(X_{1 k}\right) \leq u_{1}\right\}} \mathbb{1}_{\left\{F_{2 n}\left(X_{2 k}\right) \leq u_{2}\right\}}, \quad\left(u_{1}, u_{2}\right) \in I,
$$

and $F_{j n}(t):=\frac{1}{n} \sum_{k=1}^{n} \mathbb{1}_{]-\infty, t]}\left(X_{j k}\right), j=1,2$. In order to estimate the divergences $D_{\phi}\left(\theta, \theta_{T}\right)$ for a given $\theta \in \Theta$ in particular for $\theta=\theta_{0}$, and the parameter $\theta_{T}$, we will make use of the dual representation of $\phi$-divergences obtained by [3] Theorem 4.4. By this, we readily obtain that $D_{\phi}\left(\theta_{0}, \theta_{T}\right)$ can be rewritten into

$$
D_{\phi}\left(\theta_{0}, \theta_{T}\right):=\sup _{f \in \mathscr{F}}\left\{\int_{I} f d C_{\theta_{0}}-\int_{I} \phi^{*}(f) d C_{\theta_{T}}\right\}
$$

where $\phi^{*}$ is used to denote the convex conjugate of $\phi$, namely, the function defined by

$$
\phi^{*}: t \in \mathbb{R} \mapsto \phi^{*}(t):=\sup _{x \in \mathbb{R}}\{t x-\phi(x)\},
$$

and $\mathscr{F}$ is an arbitrary class of measurable functions fulfilling the following two conditions : $\forall f$ $\in \mathscr{F}, \int|f| d C_{\theta_{0}}$ is finite and $\phi^{\prime}\left(d C_{\theta_{0}} / d C_{\theta_{T}}\right) \in \mathscr{F}$. Furthermore, the sup in the above display is unique and is achieved at $f=\phi^{\prime}\left(d C_{\theta_{0}} / d C_{\theta_{T}}\right)$. Note that the plug-in estimate $\int_{I} \phi\left(d C_{\theta} / d C_{n}\right)$ $d C_{n}\left(u_{1}, u_{2}\right)$ of $D_{\phi}\left(\theta, \theta_{T}\right)$ is not well defined since $C_{\theta}(\cdot, \cdot)$ is not absolutely continuous with respect to $C_{n}(\cdot, \cdot)$; the use of the dual representation, as we will show, avoids this problem. By the above statement, taking the class of functions $\mathscr{F}=\left\{u \in I \mapsto \phi^{\prime}\left(1 / c_{\theta}\right) ; \theta \in \Theta\right\}$, we obtain the formula

$$
D_{\phi}\left(\theta_{0}, \theta_{T}\right)=\sup _{\theta \in \Theta}\left\{\int_{I} \phi^{\prime}\left(\frac{1}{c_{\theta}}\right) d u_{1} d u_{2}-\int_{I}\left[\frac{1}{c_{\theta}} \phi^{\prime}\left(\frac{1}{c_{\theta}}\right)-\phi\left(\frac{1}{c_{\theta}}\right)\right] d C_{\theta_{T}}\left(u_{1}, u_{2}\right)\right\},
$$

whenever $\int_{I}\left|\phi^{\prime}\left(1 / c_{\theta}\right)\right| d u_{1} d u_{2}$ is finite for all $\theta \in \Theta$. Furthermore, the sup is unique and reached at $\theta=\theta_{T}$. Hence, the divergence $D_{\phi}\left(\theta_{0}, \theta_{T}\right)$ and the parameter $\theta_{T}$ can be estimated respectively by

$$
\sup _{\theta \in \Theta}\left\{\int_{I} \phi^{\prime}\left(\frac{1}{c_{\theta}}\right) d u_{1} d u_{2}-\int_{I}\left[\frac{1}{c_{\theta}} \phi^{\prime}\left(\frac{1}{c_{\theta}}\right)-\phi\left(\frac{1}{c_{\theta}}\right)\right] d C_{n}\left(u_{1}, u_{2}\right)\right\}
$$


and

$$
\arg \sup _{\theta \in \Theta}\left\{\int_{I} \phi^{\prime}\left(\frac{1}{c_{\theta}}\right) d u_{1} d u_{2}-\int_{I}\left[\frac{1}{c_{\theta}} \phi^{\prime}\left(\frac{1}{c_{\theta}}\right)-\phi\left(\frac{1}{c_{\theta}}\right)\right] d C_{n}\left(u_{1}, u_{2}\right)\right\},
$$

in which $C_{\theta_{T}}$ is replaced by $C_{n}$. Note that this class of estimates contains the maximum pseudo-likelihood (MPL) estimator proposed by [12] ; it is obtained for the $K L_{m}$-divergence taking $\phi(x)=-\log (x)+x-1$. The results in [1] show that, for $\Theta=\left[\theta_{0}, \infty\right)$, and when the true value $\theta_{T}$ of the parameter is equal to $\theta_{0}$ the classical asymptotic normality property of the MPL estimate is no more satisfied. To circumvent this difficulty, in what follows, we enlarge the parameter space $\Theta$ into a wider space $\Theta_{e} \supset \Theta$. This is tailored to let $\theta_{0}$ become an interior point of $\Theta_{e}$. More precisely, set

$$
\Theta_{e}:=\left\{\theta \in \mathbb{R}^{d} \text { such that } \int\left|\phi^{\prime}\left(1 / c_{\theta}\left(u_{1}, u_{2}\right)\right)\right| d u_{1} d u_{2}<\infty\right\} .
$$

Assume that $\stackrel{\Theta}{e}_{e}$ is non empty set and $\Theta_{e} \supset \Theta$. So, applying (3), with the class of functions

$$
\mathscr{F}:=\left\{\left(u_{1}, u_{2}\right) \mapsto \phi^{\prime}\left(1 / c_{\theta}\left(u_{1}, u_{2}\right)\right) ; \theta \in \Theta_{e}\right\},
$$

we obtain

$$
D_{\phi}\left(\theta_{0}, \theta_{T}\right)=\sup _{\theta \in \Theta_{e}}\left\{\int_{I} \phi^{\prime}\left(\frac{1}{c_{\theta}}\right) d u_{1} d u_{2}-\int_{I}\left[\frac{1}{c_{\theta}} \phi^{\prime}\left(\frac{1}{c_{\theta}}\right)-\phi\left(\frac{1}{c_{\theta}}\right)\right] d C_{\theta_{T}}\left(u_{1}, u_{2}\right)\right\} .
$$

Furthermore, the sup in this display is unique and reached in $\theta=\theta_{T}$. Hence, we propose to estimate $D_{\phi}\left(\theta_{0}, \theta_{T}\right)$ by

$$
\widehat{D_{\phi}}\left(\theta_{0}, \theta_{T}\right):=\sup _{\theta \in \Theta_{e}} \int_{I} m\left(\theta, u_{1}, u_{2}\right) d C_{n}\left(u_{1}, u_{2}\right)
$$

and to estimate the parameter $\theta_{T}$ by

$$
\widehat{\theta}_{n}:=\arg \sup _{\theta \in \Theta_{e}}\left\{\int_{I} m\left(\theta, u_{1}, u_{2}\right) d C_{n}\left(u_{1}, u_{2}\right)\right\},
$$

where $m\left(\theta, u_{1}, u_{2}\right)$ is equal to

$$
\int_{I} \phi^{\prime}\left(\frac{1}{c_{\theta}\left(u_{1}, u_{2}\right)}\right) d u_{1} d u_{2}-\left\{\phi^{\prime}\left(\frac{1}{c_{\theta}\left(u_{1}, u_{2}\right)}\right) \frac{1}{c_{\theta}\left(u_{1}, u_{2}\right)}-\phi\left(\frac{1}{c_{\theta}\left(u_{1}, u_{2}\right)}\right)\right\} .
$$

In the sequel, we denote by $\frac{\partial}{\partial \theta} m\left(\theta, u_{1}, u_{2}\right)$ the $d$-dimensional vector with entries $\frac{\partial}{\partial \theta_{i}} m\left(\theta, u_{1}, u_{2}\right)$ and $\frac{\partial^{2}}{\partial \theta^{2}} m\left(\theta, u_{1}, u_{2}\right)$ the $d \times d$-matrix with entries $\frac{\partial^{2}}{\partial \theta_{i} \partial \theta_{j}} m\left(\theta, u_{1}, u_{2}\right)$.

\section{The asymptotic behavior of the estimates}

In this section, we provide both weak and strong consistency of the estimates (10). We also state their asymptotic normality and evaluate their limiting variance. We will use the following notations

$$
K_{1}\left(\theta, u_{1}, u_{2}\right):=\phi^{\prime}\left(\frac{1}{c_{\theta}\left(u_{1}, u_{2}\right)}\right)
$$

and

$$
K_{2}\left(\theta, u_{1}, u_{2}\right):=\left\{\phi^{\prime}\left(\frac{1}{c_{\theta}\left(u_{1}, u_{2}\right)}\right) \frac{1}{c_{\theta}\left(u_{1}, u_{2}\right)}-\phi\left(\frac{1}{c_{\theta}\left(u_{1}, u_{2}\right)}\right)\right\} .
$$

Let $\mathscr{Q}$ be the set of u-shaped functions, and $\mathscr{R}$ the set of reproducing u-shaped functions (see e.g. [13] p. 894 for definition). We make use of the following conditions. 
(C.1) There exists a neighborhood $N\left(\theta_{T}\right) \subset \Theta_{e}$ of $\theta_{T}$ such that the first and the second partial derivatives with respect to $\theta$ of $K_{1}\left(\theta, u_{1}, u_{2}\right)$ are dominated on $N\left(\theta_{T}\right)$ by some $\lambda$-integrable functions ;

(C.2) There exists a neighborhood $N\left(\theta_{T}\right)$ of $\theta_{T}$, such that for all $\theta \in N\left(\theta_{T}\right)$, the function $\frac{\partial}{\partial \theta} m\left(\theta, u_{1}, u_{2}\right):(0,1)^{2} \rightarrow \mathbb{R}$ is continuously differentiable and there exist functions $r_{i} \in \mathscr{R}, \widetilde{r}_{i} \in \mathscr{R}$ and $q \in \mathscr{Q}\left(i, j=1,2, \quad i \neq j\right.$ and $\left.\ell, \ell^{\prime}=1, \ldots, m\right)$ with

(i) $\left|\frac{\partial}{\partial \theta_{\ell}} m\left(\theta, u_{1}, u_{2}\right)\right| \leq r_{1}\left(u_{1}\right) r_{2}\left(u_{2}\right),\left|\frac{\partial^{2}}{\partial \theta_{\ell} \partial u_{i}} m\left(\theta, u_{1}, u_{2}\right)\right| \leq \widetilde{r}_{i}\left(u_{i}\right) r_{j}\left(u_{j}\right)$;

(ii) $\left|m^{2}\left(\theta, u_{1}, u_{2}\right)\right| \leq \widetilde{r}_{i}\left(u_{i}\right) r_{j}\left(u_{j}\right)$;

(iii) $\left|\frac{\partial^{3}}{\partial \theta^{3}} K_{2}\left(\theta, u_{1}, u_{2}\right)\right| \leq \widetilde{r}_{i}\left(u_{i}\right) r_{j}\left(u_{j}\right)$;

(iv) $\left|m\left(\theta, u_{1}, u_{2}\right)\right| \leq \widetilde{r}_{i}\left(u_{i}\right) r_{j}\left(u_{j}\right)$;

(v) $\left|\frac{\partial}{\partial \theta_{\ell}} m\left(\theta, u_{1}, u_{2}\right)\right|^{2} \leq \widetilde{r}_{i}\left(u_{i}\right) r_{j}\left(u_{j}\right),\left|\frac{\partial^{2}}{\partial \theta_{\ell} \partial \theta_{\ell^{\prime}}} m\left(\theta, u_{1}, u_{2}\right)\right| \leq \widetilde{r}_{i}\left(u_{i}\right) r_{j}\left(u_{j}\right)$;

(vi) $\left|\frac{\partial}{\partial u_{i}} m\left(\theta, u_{1}, u_{2}\right)\right| \leq \widetilde{r}_{i}\left(u_{i}\right) r_{j}\left(u_{j}\right)$

and $\int_{I}\left\{r_{1}\left(u_{1}\right) r_{2}\left(u_{2}\right)\right\}^{2} d C_{\theta_{T}}\left(u_{1}, u_{2}\right)<\infty, \int_{I}\left\{q_{i}\left(u_{i}\right) \widetilde{r}_{i}\left(u_{i}\right) r_{j}\left(u_{j}\right)\right\} d C_{\theta_{T}}\left(u_{1}, u_{2}\right)<\infty$;

(C.3) The matrix $\int\left(\partial^{2} / \partial^{2} \theta\right) m\left(\theta, u_{1}, u_{2}\right) d C_{\theta_{T}}\left(u_{1}, u_{2}\right)$ is non singular;

(C.4) The function $\left(u_{1}, u_{2}\right) \in I \mapsto \frac{\partial}{\partial \theta} m\left(\theta_{T}, u_{1}, u_{2}\right)$ is of bounded variation on $I$.

Theorem 3.1 Assume that conditions (C.1-3) hold.

1. Let $B\left(\theta_{T}, n^{-1 / 3}\right):=\left\{\theta \in \Theta_{e},\left|\theta-\theta_{T}\right| \leq n^{-1 / 3}\right\}$, then as $n$ tends to infinity, with probability one, the function $\theta \mapsto \int m\left(\theta, u_{1}, u_{2}\right) d C_{n}\left(u_{1}, u_{2}\right)$ attains its maximum value at some point $\widehat{\theta}_{n}$ in the interior of $B\left(\theta_{T}, n^{-1 / 3}\right)$, which implies that the estimate $\widehat{\theta}_{n}$ is consistent almost surely and satisfies $\int \frac{\partial}{\partial \theta} m\left(\widehat{\theta}_{n}, u_{1}, u_{2}\right) d C_{n}\left(u_{1}, u_{2}\right)=0$.

2. $\sqrt{n}\left(\widehat{\theta}_{n}-\theta\right)$ converges in distribution to a centered multivariate normal random variable with covariance matrix

$$
\begin{aligned}
\boldsymbol{\Xi}_{\phi} & =\mathbf{S}^{-1} \mathbf{M S}^{-1}, \\
\text { with } \mathbf{S} & :=-\int \frac{\partial^{2}}{\partial \theta^{2}} m\left(\theta_{T}, u_{1}, u_{2}\right) d C_{\theta_{T}}\left(u_{1}, u_{2}\right), \\
\text { and } \mathbf{M} & :=\operatorname{Var}\left[\frac{\partial}{\partial \theta} m\left(\theta_{T}, F_{1}\left(X_{1}\right), F_{2}\left(X_{2}\right)\right)+W_{1}\left(\theta_{T}, X_{1}\right)+W_{2}\left(\theta_{T}, X_{2}\right)\right], \\
\text { where } W_{i}\left(\theta_{T}, X_{i}\right) & :=\int_{I} \mathbb{1}_{\left\{F_{i}\left(X_{i}\right) \leq u_{i}\right\}} \frac{\partial^{2}}{\partial \theta \partial u_{i}} m\left(\theta_{T}, u_{1}, u_{2}\right) c_{\theta_{T}}\left(u_{1}, u_{2}\right) d u_{1} d u_{2}, i=1,2 .
\end{aligned}
$$

\section{New tests of independence}

In the framework of the parametric copula model, the null hypothesis, i.e., the independence case $C_{\theta}\left(u_{1}, u_{2}\right)=u_{1} u_{2}$ corresponds to $\mathscr{H}_{0}: \theta_{T}=\theta_{0}$. We consider the composite alternative hypothesis $\mathscr{H}_{1}: \theta_{T} \neq \theta_{0}$. Since, $\theta_{0}$ is a boundary value of the parameter space $\Theta$, we can see that the convergence in distribution of the corresponding pseudo-likelihood ratio statistic to a $\chi^{2}$ random variable does not hold; see [1]. We give now a solution to this problem. We propose the following statistics

$$
\mathbf{T}_{n}:=\frac{2 n}{\phi^{\prime \prime}(1)} \widehat{D_{\phi}}\left(\theta_{0}, \theta_{T}\right)
$$

We will use the following additional conditions 
(C.5) We have

$$
\lim _{\theta \rightarrow \theta_{0}} \frac{\partial^{2}}{\partial \theta_{\ell} \partial u_{i}} m\left(\theta, u_{1}, u_{2}\right)=0,
$$

and there exist $M_{1}>0$ and $\delta_{1}>0$ such that, for all $\theta$ in some neighborhood of $\theta_{0}$, one has, for $i=1,2$,

$$
\left|\frac{\partial^{2}}{\partial \theta_{\ell} \partial u_{i}} m\left(\theta, u_{1}, u_{2}\right) c_{\theta_{T}}\left(u_{1}, u_{2}\right)\right|<M_{1} r\left(u_{i}\right)^{-1.5+\delta_{1}} r\left(u_{3-i}\right)^{0.5+\delta_{1}},
$$

where $r(u):=u(1-u)$ for $u \in(0,1)$.

Theorem 4.1 (1) Assume that conditions (C.1-5) hold. If $\theta_{T}=\theta_{0}$, then the statistic $\mathbf{T}_{n}$ converges in distribution to a $\chi^{2}$ variable with d degrees of freedom.

(2) Assume that conditions (C.1-4) hold. If $\theta_{T} \neq \theta_{0}$, then $\sqrt{n}\left(\widehat{D_{\phi}}\left(\theta_{0}, \theta_{T}\right)-D_{\phi}\left(\theta_{0}, \theta_{T}\right)\right)$ converges in distribution to a centered normal variable with variance

$$
\sigma_{\phi}^{2}\left(\theta_{0}, \theta_{T}\right):=\operatorname{Var}\left[m\left(\theta_{T}, F_{1}\left(X_{1}\right), F_{2}\left(X_{2}\right)\right)+Y_{1}\left(\theta_{T}, X_{1}\right)+Y_{2}\left(\theta_{T}, X_{2}\right)\right],
$$

where

$$
Y_{i}\left(\theta_{T}, X_{i}\right):=\int_{I} \mathbb{1}_{\left\{F_{i}\left(X_{i}\right) \leq u_{i}\right\}} \frac{\partial}{\partial u_{i}} m\left(\theta_{T}, u_{1}, u_{2}\right) c_{\theta_{T}}\left(u_{1}, u_{2}\right) d u_{1} d u_{2}, \quad i=1,2 .
$$

Remark 1 The above regularity conditions are satisfied by a large number of parametric families of bivariate copulas; see for instance [15].

Remark 2 The parameters (11) and (11) may be consistently estimated respectively by the sample mean of

$$
\frac{\partial^{2}}{\partial \theta^{2}} m\left(\widehat{\theta}_{n}, F_{1 n}\left(X_{1, k}\right), F_{2 n}\left(X_{2, k}\right)\right), \quad k=1, \ldots, n,
$$

and the sample variance of

$$
\frac{\partial}{\partial \theta} m\left(\widehat{\theta}_{n}, F_{1 n}\left(X_{1, k}\right), F_{2 n}\left(X_{2, k}\right)\right)+W_{1}\left(\widehat{\theta}_{n}, X_{1, k}\right)+W_{2}\left(\widehat{\theta}_{n}, X_{2, k}\right), \quad k=1, \ldots, n,
$$

as was done in [7]. The asymptotic variance (12) can be consistently estimated in the same way.

\section{Concluding remarks}

We have introduced a new estimation and test procedure in parametric copula models with unknown margins. The methods is based on divergences between copulas and the duality technique. It generalizes the maximum pseudo-likelihood one, and applies both when the parameter is an interior or a boundary value, in particular for testing the null hypothesis of independence. It will be interesting to investigate the problem of the choice of the divergence which leads to an "optimal" (in some sense) estimate or test in terms of efficiency and robustness.

\section{Références}

[1] S. Bouzebda and A. Keziou. A test of independence in some copula models. Math. Methods Statist., 17(2) :123-137, 2008. 
[2] Michel Broniatowski and Amor Keziou. Parametric estimation and tests through divergences and the duality technique. Journal of Multivariate Analysis (2008), doi :10.1016/j.jmva.2008.03.011.

[3] Michel Broniatowski and Amor Keziou. Minimization of $\phi$-divergences on sets of signed measures. Studia Sci. Math. Hungar., 43(4) :403-442, 2006.

[4] Paul Deheuvels. Propriétés d'existence et propriétés topologiques des fonctions de dépendance avec applications à la convergence des types pour des lois multivariées. $C$. R. Acad. Sci. Paris Sér. A-B, 288(2) :A145-A148, 1979.

[5] Paul Deheuvels. Nonparametric test of independence. In Nonparametric asymptotic statistics (Proc. Conf., Rouen, 1979) (French), volume 821 of Lecture Notes in Math., pages 95-107. Springer, Berlin, 1980.

[6] Paul Deheuvels. A Kolmogorov-Smirnov type test for independence and multivariate samples. Rev. Roumaine Math. Pures Appl., 26(2) :213-226, 1981.

[7] C. Genest, K. Ghoudi, and L.-P. Rivest. A semiparametric estimation procedure of dependence parameters in multivariate families of distributions. Biometrika, 82(3) :543$552,1995$.

[8] Harry Joe. Parametric families of multivariate distributions with given margins. $J$. Multivariate Anal., 46(2) :262-282, 1993.

[9] Harry Joe. Multivariate models and dependence concepts, volume 73 of Monographs on Statistics and Applied Probability. Chapman \& Hall, London, 1997.

[10] Friedrich Liese and Igor Vajda. Convex statistical distances, volume 95. BSB B. G. Teubner Verlagsgesellschaft, Leipzig, 1987.

[11] Roger B. Nelsen. An introduction to copulas, volume 139 of Lecture Notes in Statistics. Springer-Verlag, New York, 1999.

[12] David Oakes. Multivariate survival distributions. J. Nonparametr. Statist., 3(3-4) :343354, 1994.

[13] F. H. Ruymgaart. Asymptotic normality of nonparametric tests for independence. Ann. Statist., 2 :892-910, 1974.

[14] M. Sklar. Fonctions de répartition à $n$ dimensions et leurs marges. Publ. Inst. Statist. Univ. Paris, 8 :229-231, 1959.

[15] Hideatsu Tsukahara. Semiparametric estimation in copula models. Canad. J. Statist., 33(3) :357-375, 2005.

[16] Weijing Wang and A. Adam Ding. On assessing the association for bivariate current status data. Biometrika, 87(4) :879-893, 2000. 